\title{
CALCUL INTERPRÉTATIF MÉTAPHORIQUE À L'EXEMPLE DE LA STRUCTURE LE N1 EST UN N2
}

\begin{abstract}
Domańska-Gruszka Jolanta, Calcul interprétatif métaphorique à l'exemple de la structure "Le N1 est un $\mathrm{N} 2$ " [Interpretative metaphoric calculation on the example of the structure of le N] and un N2]. Studia Romanica Posnaniensia, Adam Mickiewicz University Press, Poznań, vol. XXXIII : 2006, pp. 105-112. ISBN 83-232-1643-6, ISSN 0137-2475.

In the present paper the author tries to analyze the phenomenon of linguistic metaphor not at the level of 'languc' but rather as the result of discursive uses. The basic predicative structurc le N1 est un N2 in French in metaphorical use is treated not as categorization but as characierization of subject. The author compares pragma-semantic and discursive approach of the metaphorical predication and its interpretation - analogy as included in the system and 'conformity judgment' as a local characterization. The final considerations are about philosophical implications of metaphorical words uses.
\end{abstract}

Dans le présent article nous pensons montrer dans quelle mesure les approches discursives en linguistique et philosophiques sont fructueuses et inspiratrices pour les études métaphorologiques (la quantité de travaux consacrés à la reine des figures rhétoriques autorisant l'emploi de ce qualifiatif).

Du point de vue sémiotique il est intenable d'envisager la métaphore comme " substitution " d'un lexème à un autre (comme l'envisageaient les grammairiensrhétoricien classiques, Dumarsais et Fontannier). Le traitement en termes de selection des traits ou de sèmes (Groupe $\mu$ de Liège, F. Rastier) s'avère efficace pour le traitement des métaphores lexicalisées (ou métaphoriquement parlant mortes), celles qui figurent dans des dictionnaires avec mention par anal., par ext., fig., par metaph., auquel cas la signification des divers acceptions (métaphoriques) d'un lexème est stabilisé à tel point qu'on en peut définir une collection / hiérarchie des sèmes.

Dans une perspective disursive le mot (vocable) est intéressant en tant qu'il est pourvu d'un sens précis, d'une référence actuelle. L'unicité du signe lexical n'est même plus posé car le sens se construit dans un contexte particulier qui filtre les valeurs virtuelles d'un lexème et en peut susciter de nouvelles. Nous sommes d'avis que la métaphore gagne à être interprétée en termes discursifs, comme un procès dynamique de création de sens, accompagné d'un calcul interpétatif spécifique. 
Le domaine d'activité, le champs d'application du transfert métaphorique est tellement vaste et stratifié (rien qu'en termes de parties du discours, la métaphore peut arriver à un nom un tendre arbrisseau pour désigner un enfant, à un verbe Des orteils riaient, à un participe un sourire barricadé, à un adverbe Le silence bourdonnait électriquement) qu'en proposer une définition unitaire et globale est pratiquement impossible.

Une définition générale et acceptaple pour des chercheurs de tous horizons peut être celle proposée par Prandi (Prandi, 1999). Selon cet auteur la métaphore constitue « la projection d'une entité ou d'une connexion entre unités dans un domaine étranger » (p. 192). Définition suffisamment générale pour être applicable à la majorité des énoncés métaphoriques mais plutôt inopérante pour expliquer leur spécifcité. Une autre tentative d'une vision globale du processus métaphorique en termes sémantico-référentiels qui va retenir notre attention est celle de G. Kleiber (Kleiber, 1999). Selon lui la structure typique (prototypique?) de l'énoncé métaphorique est celle de la prédication d'appartenance Le N1 est un N2 et c'est cette structure que nous alons prendre en considérations dans la suite de notre investigation sur l'interpétation de la métaphore. La proposition de traiter les prédications métaphoriques comme " jugements de conformité » de P. Cadiot (Cadiot, 2002) comme une issue situationnelle et discursive dans l'interprétation des prédications nominales métaphoriques consituera l'aboutissement de notre investigation avec une brève intrusion philosophique.

\section{LE DÉNOMINATEUR COMMUN DES MÉTAPHORES OU LA CATÉGORISATION INDUE}

Toute réflexion sur la métaphore doit fournir une réponse à deux questions : 1) qu'est-ce qui permet de reconnaître la métaphore dans un texte / situation? 2) une fois la figure identifiée, quel est le processus interprétatif approprié ?

Selon Kleiber (Kleiber, 1999) à l'origine de la déviance (critère identificatoire) métaphorique se trouve le délit de catégorisation' - " il s'agit de l'emploi d'une catégorie lexicale pour une occurence (ou référent) qui normalement (ou littéralement) ne peut pas être rangé dans cette catégorie lexicale ou, si l'on préfère, ne peut pas être dénommé ainsi » (Kleiber, 1999, p. 125). Ainsi la reconnaissance de la métaphore doit passer par la validation référentielle. Cette définition sémantico-pragmatique s'applique à toutes les occurences de l'emploi métaphorique des lexèmes :

- la forme dite canonique de la métaphore nominale prédicative du type le $\mathrm{NI}$ est un $\mathrm{N} 2$,

${ }^{1}$ On constatc aussi la catégorisation indue dans le cas de la métonymie et synecdoque il épousa une grosse dot; on trouvera in Kleiber (1999) une description complexe et complète de la structure et de l'interprétation différentes des ces trois figures. 
- le désignateur métaphorique Regarde la giraffe ! (dans le cas ou l'on désigne un être humain),

- les métaphores verbales Le soleil nous a souri et adjectivales lodowata cisza où nous avons à chaque fois affaire à une sorte de méprise (intentionelle) catégorielle.

Dans une prédication métaphorique comme cette fille est un bouleau (Groupe $\mu, 1970$ ) la catégorie lexicale bouleau est employée en dehors de son aire de catégorisation (une espèce d'arbre) à l'occurence d'une autre catégorie lexicalisée fille. Dans la métaphore adjectivale lodowata cisza l'adjectif ne renvoie pas à la qualité qu'il dénote en vertu de son sens.

Kleiber et Prandi postulent d'étendre le critère de la déviance de catégorisation aussi aux métaphores verbales et adjectivales.

Dans la métaphore verbale Natalia plynie qui réfère à la manière de marcher d'une (jeune) femme, l'action accomplie par le sujet ne correspond pas à celle qui est dénoté par le verbe plynie, le verbe métaphorique catégorise l'occurence de marcher comme étant celle de nager. Ici la déviance n'est évidente qu'au niveau référentiel, la phrase hors contexte étant sémantiquement non déviante.

Le même raisonnement est valable dans le cas des métaphores présentant la violation des contraintes selectionnelles (traité d'incongruité ou incongruence par Kleiber) et pour lesquelles il est impossible de trouver " le double virtuel disponible " (Prandi 1992) ou qui dénotent l'occurence d'une catégorie verbale ou adjectivale qui n'est pas lexicalisée dans une langue. Dans la métaphore La nuit rêve le verbe métaphorique rêver dénote une activité de la lune qui n'est pas catégorisée en français mais qui n'est sûrement pas celle que dénote le verbe rêver.

La déviance sémantico-référentielle commune à tous les types de métaphores est une déviance de catégorisation : un terme ou une catégorie lexicale est employé pour une occurence qui ne fait a priori ou littéralement pas partie de sa catégorie (Kleiber, 1999).

\section{CATÉGORISATION INDUE - MÉTAPHORE - ANALOGIE - INTERPRÉTATION}

Dans ce qui précède nous avons essayé de fournir une réponse à la première question identificatoire de la métaphore : qu'est-ce qui permet de reconnaître la métaphore dans un texte / situation? La question suivante est celle de la nature du processus interprétatif de la métaphore ainsi que de son statut linguistique.

\section{Métaphore nominale prédicative}

Selon Kleiber, le processus interprétatif mis en jeu par ma métaphore prédicative d'appartenance ${ }^{2}$ le $\mathrm{N} 1$ est un $\mathrm{N} 2$ est logiquement contenu dans cette structure

\footnotetext{
${ }^{2}$ Il ne faut pas se méprendre sur le dénomination car, pour une prédication métaphorique, il ne s'agit pas de prédicattion d'appartenance ! Seule le structure syntaxique est celle de la prédication d'appartenance.
} 
même. Une entité déjà catégorisée (ou lexicalisée) N1 se trouve rangée dans une autre catégorie $N 2$. L'interprétation de cette structure sémantico-syntaxique prédicative d'appartenance, qui relie deux lexèmes / catégories (le / les Nl est / sont un / des N2) ou une occurence d'une catégorie et une catégorie (ce / ces N1 est / sont un/des N2) ${ }^{3}$ inpose une interprétation qui, selon Kleiber, est celle de trouver les raisons de ranger le / ce NI dans la catégorie de un N2. Or, cette opération de placer ou de ranger le / ce NI dans un N2 n'est rien d'autre que la recherche d'analogie / ressemblance entre les deux lexèmes soit en termes de traits nécessaires et suffisants soit en termes de proximité/périphérie par rapport à un prototype.

Dans le cas de la prédication métaphorique cette opération d'appariement entre le $N 1$ et un N2 n'est ni inscrite dans la langue ni ne reflète l'ordre conceptuel préalable. La catégorisation est alors une catégorisation défective, indue et l'on est obligé de procéder à un calcul interprétatif d'analogie / ressemblance pour justifier la classification non standard. L'énoncé de ressemblance est une étape dans le processus de la compréhension de la métaphore. Cette interprétation n'est pas de nature sémantique - il n'est pas question pour un terme métaphorique d'un changement de sens - dans la métaphore cette jeune fille est un bouleau la catégorie lexicale bouleau ne change pas de sens. Or, l'interprétation métaphorique ou le sens figuré n'a pas un statut sémantique mais il s'agit d'un sens construit, inféré à partir du sens littéral. Autrement dit, le constat d'un défait de catégorisation est une première instruction de sens qui pousse le récepteur à chercher un sens cohérent.

G. Kleiber (Kleiber, 1999) refuse toute analyse sémantique de l'interprétation métaphorique «Un énoncé métaphorique n'a qu'un sens, un sens littéral noncomparatif. Ce qu'on appelle l'interprétation métaphorique ou le sens figuré ou sens métaphorique ou tropique n'a pas un statut sémantique ». A l'origine ce cette constatation se trouve la thèse de la double nature du sens qui peut être a) donné $a$ priori, par la combinaison des sens codés, associés aux formes linguistiques ou b) construit, inféré à partir du sens stable, littéral. La tâche principale est désormais de tracer le parcours interprétatif pertinent pour ce type d'énoncés conflictuels qui, à partir du sens littéral, mènera à l'interprétation métaphorique et non pas à un sens métaphorique.

La métaphore, traité au niveau logique/conceptuel comme la catégorisation plaçant une occurence dans une catégorie (ou concept) qui n'est pas la sienne, prend la forme syntaxique de la structure d'appartenance ou d'inclusion

\section{SN1 est un / du N2}

où c'est l'article indéfini ou partitif qui indique qu'il s'agit d'une catégorisation d'une occurence. L'opération de catégorisation consiste en un appariement entre les traits ou la définition de la catégorie lexicale (N2) et l'occurence (N1). C'est ainsi

${ }^{3}$ Sur la relation entre la structure syntaxique canonique le N1 est un N2 et son interprétation comme phrase classifictoire ou prédicative d'appartenance la femme est une fleur, voir Tamba (1999). 
que l'on revient à l'éternelle analogie ou ressemblance, laquelle depuis Aristote était considérée comme le pivot de la métaphore.

Que l'on parle de conditions nécessaires et suffisantes ou de prototype, qu'elle soit faite analytiquement ou procéduralement, la catégorisation est une comparaison entre, si l'on veut, le modèle que l'on a de l'occurence et celui de la catégorie, comparaison qui s'abolit en cas de réussite, l'occurence étant placée sans problème dans la catégorie en question. (...) Dans le cas de la métaphorc, c'est la même opération d'apparicment qui se trouve déclenchée par la carégorisation effectuée, mais comme il s'agit d'une catégorisation que l'on sait indue dès le départ, cette opération n'est pas routinière, automatique, mais c'est un véritable calcul interprétatif auquel on est convié et qui conduit à chercher des traits qui justifient la catégorisation et qui ne peuvent être ceux de la catégorisation standard. Le résultat ne peut être que la similitude, l'analogie, le modèle comparatif: il faut que l'on découvre des traits communs par le fait même qu'il s'agit d'unc opération de catćgorisation, mais ces traits ne peuvent être que des traits de ressemblance, analogiques, puisqu'il s'agit d'une catégorisation indue (Kleiber, 1999, p. 132).

\section{INTERPRÉTATION DE L'ENONCÉ MÉTAPHORIQUE}

La thèse de Kleiber est celle que le parcours intreprétatif métaphorique déclanché par la catégorisation non standard est le parcours interprétatif de ressemblance / analogie contenu dans la structure même de la prédication d'appartenance le NI est un N2. Or, si on peut être d'accord avec le postulat de la méprise catégorielle comme déclancheur du processus interprétatif métaphorique, il nous paraît difficile d'admettre de chercher par une voie d'analogie les traits qui permettraient de justifier la catégorisation d'une occurence dans une catégorie qui n'est la sienne. Lorsqu'on a constaté une catégorisation indue dans Cette jeune fille est un bouleau s'agit-il effectivement d'une opération de catégorisation - peut-on prétendre ranger un individu comme une occurence de catégorie bouleau? Kleiber resout ce problème en disant qu'il s'agit plutôt d'une caractérisation du $N I$ à l'aide de certains traits spécifiques du N2. Il passe ainsi de la catégorisation à la caractérisation, qui est une opération différente. Kleiber parle de la propriété saillante générique, p.ex. l'agressivité pour la catégorie doberman ce qui permet d'interpréter un énoncé paradoxale et apparamment contradictoire Ce teckel est un doberman. Un teckel concret n'est pas rangé dans la catégorie qui n'est pas de toute évidence la sienne mais caractérisé par une propriété reconnue comme typique pour la catégorie doberman à savoir l'agressivité.

Le problème se pose alors de prévoir un répertoire de propriétés saillantes génériques pour chaque prédicat nominal, parce que le phénomène même de la métaphore prouve qu'il n'y a pas de restrictions d'emploi pour les prédicats nominaux métaphoriques. 


\section{SOLUTION DISCURSIVE}

Cadiot (Cadiot, 2002) parle, pour l'interprétation des métaphores nominales prédicatives, des jugements de conformité qui sont « sortes de caractéristiques locales, ponctuelles, contingentes (...) immédiatement prises en charge par la langue » et « prédications déliées de tout engagement ontologique » (ibid. p. 40), qui n'opèrent pas de catégorisation sensu stricto mais attribuent une caractéristique contingente. Les prédications métaphoriques sont, selon Cadiot, des jugements de conformité qui, sans prétendre catégoriser leurs sujets, les caractérisent à un certain niveau de généralité. A la différence des prédications d'appartenance qui allèguent une identité catégorielle, les prédications métaphoriques caractérisent le NI-Arsène Lupin est un voleur - prédication d'appartenance et Paul est un (vrai, sacré) voleur - jugement de conformité dans l'interpétation où l'individu en question p.ex. triche sur les salaires de ses employés -- jugement de conformité, caractéristique contingente.

\section{Cette fille est un rossignol.}

Pierre est un boucher. (dans le cas où Pierre est au vrai p.ex. un médecin)

Kurski jest bulterierem Kaczyniskich.

Ton âme est un paysage choisi.

Dans les prédications métaphoriques ci-dessus nous constatons des catégorisations indues, à l'opposé de l'identité stricte par appartenance à une catégorie (Kleiber). Quant à leur interprétation, au 'sens figuré' qu'elles engendrent, il y a lieu de parler d'une sorte de caractéristique contingente, situationelle du sujet. Pour la prédication Kurski jest bulterierem Kaczyńskich par exemple, bulterier ne désigne pas seulement une agressivité forte mais pour un interlocuteur polonais joue aussi sur une certaine ressemblance physique entre la personne en question et la race du chien. Tyvaert (Tyvaert 1985) remarque que les prédications atypiques, dont prédications métaphoriques, sont le plus souvent des occurences des structures logiques d'appartenance Le N1 est un N2 qui relient non des arguments à des classes mais des individus dans des situations pointées ${ }^{4}$. Les prédications d'appartenance, faiblement informatifs, saturées de sens, seraient de langue tandis que les prédications métaphoriques (comme jugements de confortmité) seraient de discours.

Il y a lieu de rappeler ici que la métaphore est un phénomène qui montre au mieux la nature du mot comme principe de transaction des sens avec des thématiques différentes et non une unité préétablie de sens. Ainsi dans Natalie est un rossignol le sens lexical du mot rossignol se trouve engagé dans un système des lieux communs, activés dans une occurence situationelle, qui viennent le compléter pour former un système d'implications plus au moins détérminé. Le lexème rossignol ne fait pas que désigner une catégorie concrète mais dans une situation de

${ }^{4}$ Les déterminants des $\mathrm{N} /$ sont de préference spécifiques (définis, démonstratifs ou possessifs) ou $\mathrm{Nl}$ sont des noms propres. 
discours, dans un emploi particulier, caractérise le sujet cette fille comme étant joyeuse, matinale, insouciante, ayant une belle voix (la liste n'est pas exhaustive). Cadiot parle d'un double régime des noms qui dans un emploi dénominatif désignent des objets ou bien renvoient à une propriété caractéristique dans un emploi prédicatif.

\section{LAA MÉTAPHORE - ENTRE LE LINGUISTIQUE ET LE PHILOSOPHIQUE}

Dans la sémantique linguistique classique les mots sont considérés comme des étiquettes des concepts objectifs des référents. C'est dans cette perspective que la métaphore est considéré comme «catégorisation indue 》 (Kleiber), "conflit conceptuel » (Prandi) ou «incompatibilité sémique» (Rastier). Dans une perspective sémasiologique l'emploi métaphorique des noms fait éclater l' "objet conceptualisé » de la signification lexicale. En termes phénoménologiques les objets du monde peuvent être saisis d'une façon partielle, certaines valeurs sémiotiques échappent à la structuration conceptuelle ou défient le prétendu ordre conceptuel établi ${ }^{5}$.

La métaphore nominale est capable de rendre des caractéristiques ou des sensations phénoménologiques diverses de façon la plus pértinente et la plus adéquate, Cadiot cite comme exemple, entre autres "pluie» pour sensation / conscience de 'intesité fine et régulière' - pluie d'insultes, "tempête » pour 'chaos intempestif" - tempête hormonale ou «océan » pour 'sérénité sans limite' - océan de fraicheur.

Il serait éminement intéressant de développer cet alphabet épistémologique des sensations désignés par des métaphores, aussi dans une perspective comparative.

La métaphore prédicative qui emprunte le schéma syntaxique de la prédication d'appartenance Le N1 est un N2 fait éclater les signification établies en langue pour les ouvrir à d'autres valeurs en discours, tel caratérisation, tels lieux communs codés dans une culture (bouleau, rossignol) tel valeurs situationels et occasionels (bulterier Kaczyńskich).

' "Quant un philosophe contemporain parle de 'philosophic crépusculaires' ou 'matinales', la signification des adjcctifs cmployés ne remonte pas nécessairement à nos expérience météorologiques. Il est bien plus probable que nos expériences du matin et du soir plongent dans la signification que revêt pour nous l'être dans son ensemble et que la jubilation des matins comme le mystère du crépuscule y participent déjà. De sorte que philosophie matinale se dit plus authentiquement que fraîcheur matinale ! Mais les significations ne sont limitées à aucune région spéciale d'objets, ne sont privilège d'aucun contenu. Elles surgissent précisément dans la référénce des uns aux autres et (...) dans le rassemblement de l'être tout entier autour de celui qui parle ou perçoit et qui, par ailleurs, fait partie de l'être rassemblé " . E. Lévinas, 1972, Humanisme de l'autre homme, cité d'après Cadiot 2002, p. 51. 
La métaphore défie l'organisation conceptuelle du monde établie. Elle montre l'émergeance du sens en discours selon un point de vue subjectif qui va du côté des phénomènes.

La métaphore nominale montre le potentiel de la catégorie du nom qui peut non seulement désigner les concepts mais aussi caractériser les sujets et non seulement les catégoriser.

Ainsi le phénomène de la métaphore permet d'observer les intéressantes et fructueuses articulations entre les facteurs formels, grammaticaux de la langue et le domaine des concepts, d'une ontologie naturelle et même, dans une perspective philosophique, permet de réinterpréter cette ontologie.

\section{BIBLIOGRAPHIE}

Cadiot P. (2002), Métaphore prédicative nominale et motifs lexicaux, Langue française, 134.

Fontanier P. (1968), Les figures du discours, Flammarion, Paris.

Groupe $\mu$ (1970), Rhétorique générale, Laroussc, Paris.

Kleiber G. (1999), De la sémantique de la métaphore à la pragmatique de la métaphore, in:

N. Charbonnel, G. Kleiber, La métaphore entre philosophie et rhétorique, PUF, Paris.

Prand i M. (1992), Grammaire philosophique des tropes, Les Editions de Minuit, Paris.

Prandi M. (1999), Grammaire philosophique de la métaphore, in : N. Charbonnel, G. Kleiber, La métaphore, entre philosophie et rhétorigue, PUF, Paris.

Prandi M. (2002), La métaphore, de la définition à la typologie, Langue française, 134.

Rastier F. (1987), Sémantique interprétative, PUF, Paris.

Tamba I. (1999), La femme est-elle une fleur comme le bleuet est une fleur? Métaphore et classification : les structures en "Le N1 est un N2", in : N. Charbonnel, G. Kleiber, La métaphore entre philosophie et rhétorique, PUF, Paris.

Tyvaert J.E. (1985), Formalisation de la métaphore et problème du sens, Semantikos, CNRS. 